\title{
Halo Formation and Emittance Growth of Positron Beams in Plasmas
}

\author{
P. Muggli, ${ }^{1}$ B. E. Blue, ${ }^{2}$ C. E. Clayton, ${ }^{2}$ F. J. Decker, ${ }^{3}$ M. J. Hogan, ${ }^{3}$ C. Huang, ${ }^{2}$ C. Joshi, ${ }^{2}$ T. C. Katsouleas, ${ }^{1}$ W. Lu, ${ }^{2}$ \\ W. B. Mori, ${ }^{2}$ C. L. O'Connell, ${ }^{3}$ R. H. Siemann, ${ }^{3}$ D. Walz, ${ }^{3}$ and M. Zhou ${ }^{2}$ \\ ${ }^{1}$ University of Southern California, Los Angeles, California 90089, USA \\ ${ }^{2}$ University of California, Los Angeles, California 90095, USA \\ ${ }^{3}$ Stanford Linear Accelerator Center, Stanford University, Stanford, California 94309, USA
}

(Received 26 December 2007; published 29 July 2008)

\begin{abstract}
An ultrarelativistic $28.5 \mathrm{GeV}, 700$ - $\mu \mathrm{m}$-long positron bunch is focused near the entrance of a 1.4-m-long plasma with a density $n_{e}$ between $\approx 10^{13}$ and $\approx 5 \times 10^{14} \mathrm{~cm}^{-3}$. Partial neutralization of the bunch space charge by the mobile plasma electrons results in a reduction in transverse size by a factor of $\approx 3$ in the high emittance plane of the beam $\approx 1 \mathrm{~m}$ downstream from the plasma exit. As $n_{e}$ increases, the formation of a beam halo containing $\approx 40 \%$ of the total charge is observed, indicating that the plasma focusing force is nonlinear. Numerical simulations confirm these observations. The bunch with an incoming transverse size ratio of $\approx 3$ and emittance ratio of $\approx 5$ suffers emittance growth and exits the plasma with approximately equal sizes and emittances.
\end{abstract}

DOI: 10.1103/PhysRevLett.101.055001

Minimization of emittance growth and halo formation in high-intensity charged particle beams is of utmost importance for applications such as heavy ion fusion, neutron spallation sources and nuclear waste treatment, cancer therapy, high-energy physics, and advanced accelerator concepts. With ion beams, emittance growth results from the mismatch of the beam to the periodic focusing structure, from the nonlinearities associated with nonuniform space charge forces, and from microinstabilities [1]. Halo formation and the associated emittance growth in proton accelerators [2-4] and in space charge-dominated electron beams [5] are relatively well understood.

In the context of an electron-positron $\left(e^{-} / e^{+}\right)$collider, preservation of the beams' emittance along the accelerators is paramount to reaching the beam luminosity required for particle physics discoveries. The emittance is proportional to the square root of the beam's transverse temperature, and emittance growth therefore limits the ability to focus the beam to small transverse sizes. While the beam normalized emittance is in principle preserved along a conventional radio-frequency-driven accelerator, the question of emittance preservation in novel acceleration schemes, such as the beam-driven plasma wakefield accelerator (PWFA), is an open question. The PWFA has been proposed to double the energy of the $e^{-} / e^{+}$of a linear collider in short $(10-100 \mathrm{~m})$, high accelerating gradient $(\approx 10 \mathrm{GeV} / \mathrm{m})$ plasma sections [6]. Energy doubling of $42 \mathrm{GeV}$ trailing particles of an $e^{-}$bunch in $\approx 85 \mathrm{~cm}$ of plasma has been recently demonstrated in a PWFA [7].

In the PWFA driven by an $e^{-}$bunch and operating in the nonlinear blowout regime $\left(n_{b}>n_{e}\right)$ [8], the accelerated beam propagates in a pure ion column. For a beam with a density $n_{b}$ not much greater than the plasma density $n_{e}$ $\left(n_{b} \geq n_{e}\right)$, the heavy plasma ions are immobile over the time scale of a plasma period, approximately the time between the drive and the accelerated bunch. The focusing
PACS numbers: 52.40.Mj, 29.27.-a, 41.75.Ht, 52.59.Wd

force of the ion column is therefore uniform along the bunch and is linear along the bunch radius (free of geometric aberrations), and the emittance of the accelerated bunch is thus preserved $[9,10]$. For $e^{-}$beams with densities much larger than the plasma density $\left(n_{b} \gg n_{e}\right)$, motion of the plasma ions may degrade the ion column's focusing properties and lead to emittance growth [11].

The situation is different for $e^{+}$bunches propagating in plasmas. Focusing of an $e^{+}$beam by a millimeter-long, but high density, gas jet plasma has been demonstrated [12]. The time dependence of the focusing by a 1.4-m-long, but low density $\left(<4 \times 10^{12} \mathrm{~cm}^{-3}\right)$, plasma has also been studied within single, picosecond-long $e^{+}$bunches [13]. However, in these experiments the plasma density-length product was too small for the bunch to significantly evolve along the plasma, and in this respect the plasma column could be considered as a thin focusing element. In a plasma-based accelerator with multi-GeV energy gain, the plasma will be meters long with a density in the $10^{16} \mathrm{~cm}^{-3}$ range [6], and the beams will significantly evolve in their transverse dimensions. Such an evolution is well documented for the case of $e^{-}$bunches $[9,10,14,15]$. Numerical simulations indicate that the focusing force a plasma exerts on an $e^{+}$bunch is inherently nonlinear along both the bunch radius and the length $[13,16]$. The nonlinearities are the result of nonuniformities in the density of the plasma $e^{-}$that are attracted toward, flow through, and neutralize and focus the $e^{+}$ bunch. Note that the electron plasma density inside the $e^{+}$bunch may even locally exceed the bunch density. This nonlinear focusing force is expected to lead to emittance growth of the incoming beam along the plasma length. In the experiments described here, as in ion beam experiments [2-4], this growth manifests itself through the formation of a beam halo, and through the increase in beam core size as a function of the plasma density. 
In this Letter we examine for the first time both experimentally and numerically the propagation of an ultrarelativistic $e^{+}$beam in a 1.4-m-long plasma with densities in the $0.1-5 \times 10^{14} \mathrm{~cm}^{-3}$ range, appropriate for acceleration of particles in the back of the $\approx 700-\mu \mathrm{m}$-long bunch [17]. The transverse size and shape of a beam with asymmetric initial emittances in the two transverse directions are monitored $\approx 1 \mathrm{~m}$ downstream from the plasma exit using optical transition radiation (OTR). Images of the beam show that, at this location, the beam transverse size is reduced by a factor of $\approx 3$ in the plane of high emittance when the plasma is turned on. As the plasma density is increased, the beam develops a halo that contains up to $40 \%$ of the total beam charge. Numerical simulations confirm the beam size behavior as observed after the plasma, including initial size reduction in the high emittance $x$ plane, size increase in the low emittance $y$ plane, and halo formation, all as the plasma density is increased. Analysis of the beam longitudinal slice emittance from the simulation particle phase space indicates that the emittance grows quickly over the first $10 \mathrm{~cm}$ of plasma, and increases along the bunch. Simulations also show that an incoming $e^{+}$beam with emittances in the horizontal and vertical plane different by a factor of 5 exits the plasma with emittances and sizes approximately equal. There is good agreement between the beam size and halo formation predicted by the simulations and measured in the experiment. Halo formation and emittance growth are the results of the mutual interaction between the plasma $e^{-}$that focus the $e^{+}$bunch (by neutralization) and the $e^{+}$bunch that, in turns, modifies the plasma $e^{-}$density within the bunch itself.

In the experiment, the ultrarelativistic $28.5 \mathrm{GeV} e^{+}$ beam generated by the Stanford Linear Accelerator Center (SLAC) linac is delivered to the Final Focus Test beam line [18] with $\approx 1.9 \times 10^{10} e^{+} /$bunch $(\approx 3 \mathrm{nC})$. The bunch longitudinal rms length is $\approx 730 \mu \mathrm{m}(\approx 2.4 \mathrm{ps})$. The incoming invariant emittances are $\approx 50$ and $\approx 5 \times 10^{-6} \mathrm{~m} \mathrm{rad}$ in the horizontal $(x)$ and vertical $(y)$ planes, respectively. The schematic of the experiment is similar to that of Ref. [14]. The beam is focused at the entrance of a lithium vapor column. An ultraviolet (uv, $\lambda=193 \mathrm{~nm}$ ), $20 \mathrm{~ns}$ laser pulse creates the plasma by photoionization of the low ionization potential $(5.4 \mathrm{eV})$ lithium vapor contained in a heat-pipe oven $[19,20]$. The laser beam, and therefore the plasma, is made collinear with the $e^{+}$beam by reflection off a $45^{\circ}, 150-\mu \mathrm{m}$-thick glass pellicle coated for high reflectivity at $193 \mathrm{~nm}$. The laser beam is also focused along the lithium vapor column to compensate for the absorption of uv photons and thereby retain a constant $( \pm 5 \%)$ plasma density over the column length. The plasma density is obtained from the measurements of the lithium neutral density and absorbed uv energy. The measurements of the $e^{+}$characteristics with plasma $\left(n_{e}>0\right)$ are acquired with the $e^{+}$bunch traveling along the plasma $200 \mathrm{~ns}$ after the laser pulse. Every fourth event is recorded with the laser firing after the $e^{+}$bunch $\left(n_{e}=0\right)$ in order to continuously monitor the incoming beam characteristics. The plasma length is $1.4 \mathrm{~m}$, and $n_{e}$ is varied between $\approx 10^{13} \mathrm{~cm}^{-3}$ and $\approx 5 \times 10^{14} \mathrm{~cm}^{-3}$ by adjusting the laser pulse energy. The maximum ionization fraction is $\approx 13 \%$.

The backward visible OTR emitted by the bunch when traversing $25-\mu \mathrm{m}$-thick titanium foils at $45^{\circ}$ incidence, located $\approx 1 \mathrm{~m}$ upstream (downstream) from the plasma entrance (exit), is imaged onto CCD cameras. The $e^{+}$ beam suffers scattering and emittance growth when traversing the $25.4-\mu \mathrm{m}$-thick beryllium window that isolates the accelerator ultrahigh vacuum from the heat-pipe oven buffer gas, the glass pellicle reflecting the uv laser pulse, and the OTR screen located before the plasma. For a round, double-Gaussian, $25 \mu \mathrm{m}$ beam size at the plasma entrance, the invariant emittances calculated by including this scattering are $\epsilon_{N x} \approx 390 \times 10^{-6} \mathrm{~m} \mathrm{rad}$ and $\epsilon_{N y} \approx$ $80 \times 10^{-6} \mathrm{~m} \mathrm{rad}$.

Images of the $e^{+}$beam at the downstream OTR location are shown in Fig. 1(a) for the case without plasma $\left(n_{e}=0\right)$ and in Fig. 1(b) for $n_{e} \approx 0.7 \times 10^{14} \mathrm{~cm}^{-3}$. With $n_{e}=0$ the beam is elliptical at the downstream location because of the unequal incoming emittances. The transverse profiles shown in white, obtained by summing the image in the perpendicular direction, are Gaussian to close approximation. The image with $n_{e} \approx 0.7 \times 10^{14} \mathrm{~cm}^{-3}$ [Fig. 1(b)] shows that the beam size is strongly reduced in the $x$ plane (large $\epsilon_{N}$ ), while the size in the $y$ plane (small $\epsilon_{N}$ ) is approximately the same as with $n_{e}=0$. On this image the core of the beam is surrounded by a charge halo that is the source of the shoulders observed in the transverse profiles, and which is most visible in the $y$ profile.

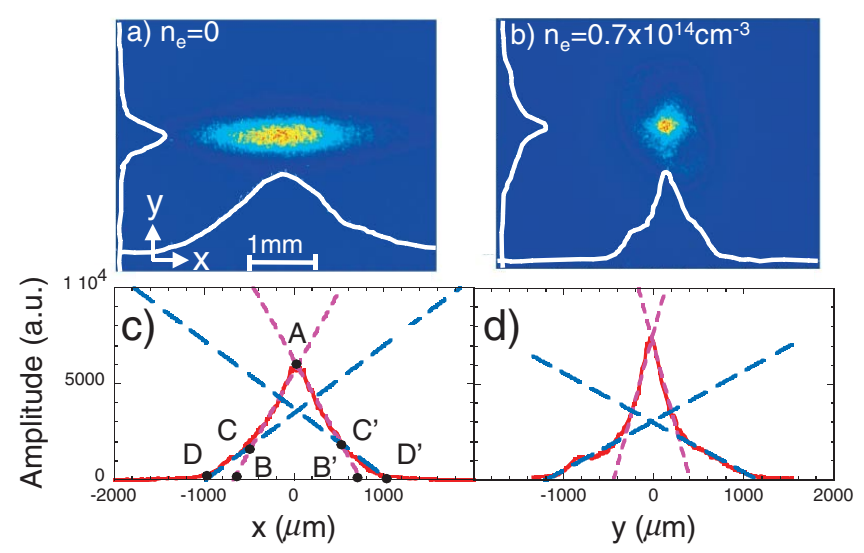

FIG. 1 (color). Experimental images of the beam at the downstream OTR location for (a) $n_{e}=0$ and (b) $n_{e} \approx 0.7 \times$ $10^{14} \mathrm{~cm}^{-3}$. Examples of the experimental beam profiles $\left(n_{e}>0\right)$, as well as the triangle fits used for the calculation of the beam transverse sizes (FWHM of $A B B^{\prime}$ triangle), and charge fractions in the core (area of $A B B^{\prime}$ triangle) and halo (area $C B D+C^{\prime} B^{\prime} D^{\prime}=2 C B D$ ) in the (c) $x$ plane and (d) $y$ plane. 
In order to quantify both the beam size and the formation of the halo as a function of $n_{e}$, the transverse beam profiles are fitted with two triangles, as shown in Figs. 1(c) and 1(d). While other methods have been used to describe beam halo formation [21,22], the method chosen here yields both the core beam size and the fractional charge contained in the core and halo. The beam size is defined as the full width at half maximum of the core triangle $\left(A B B^{\prime}\right)$. The fractional charge in the focused core and halo are defined as the area of the core triangle and two times the area of the halo triangle $(C D B)$, respectively, both divided by the total area encompassed by the triangles $\left(D C A C^{\prime} D^{\prime}\right)$. Note that with this description a Gaussian beam has about $4 \%$ of its total charge in the halo. These profiles describe well symmetric transverse profiles. Events with strong asymmetries are ignored. The resulting transverse beam sizes and charge fractions in the beam core and halo are plotted versus $n_{e}$ in Figs. 2(a) and 2(b), respectively. The important features of Fig. 2(a) are the following. First, the beam size in the $x$ plane with $n_{e}>0$ is reduced by a factor $\approx 3$ when compared to the $n_{e}=0$ case, while in the $y$ plane the beam size only increases for all $n_{e}>0$. Second, although the incoming beam has very different sizes for $n_{e}=0$ (downstream of the plasma), for $n_{e}>0$ the sizes in both planes are essentially equal and increase with $n_{e}$. Third, unlike the case of an $e^{-}$beam $[9,10,14]$, no modulation of the beam size associated with beam envelope betatron oscillation is observed with $e^{+}$in the $n_{e}$ range covered here $\left(n_{e} \geq 0.1 \times 10^{14} \mathrm{~cm}^{-3}\right)$.

Figure 2(b) shows the charge fraction contained in the core of the focused beam and in the halo for low $n_{e}$ in Fig. 2(a). In the low emittance $y$ plane the amount of charge transferred from the beam core to the halo increases linearly for $0<n_{e}<0.4 \times 10^{14} \mathrm{~cm}^{-3}$, and it remains approximately constant at $\approx 40 \%$ of the total charge at higher $n_{e}$. In the high emittance $x$ plane a smaller fraction of the charge $(\approx 25 \%)$ is transferred to the halo as soon as $n_{e}>0$, and the relative amounts of charge are essentially independent of $n_{e}\left(<2 \times 10^{14} \mathrm{~cm}^{-3}\right)$.

The propagation of the bunch in the plasma is modeled using the numerical code QUICKPIC [23]. This code uses a
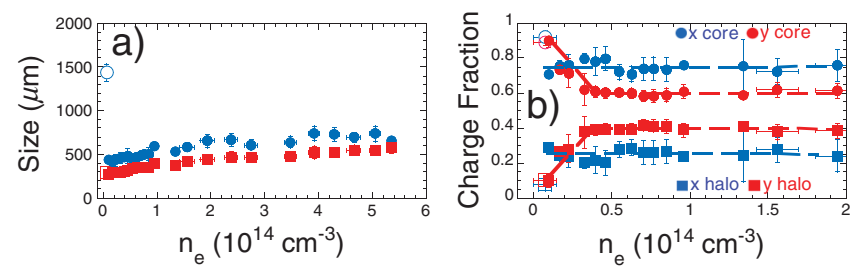

FIG. 2 (color). (a) Experimental beam transverse size and (b) fraction of the total beam charge in the beam core and the beam halo in the $x$ (blue circles) and $y$ plane (red squares) as a function of the plasma density $n_{e}$. In both figures the empty symbols are for $n_{e}=0$ and the filled symbols for $n_{e}>0$. (b) is plotted for the $n_{e}<2 \times 10^{14} \mathrm{~cm}^{-3}$ range of (a), and the dotted lines are plotted to suggest trends. quasistatic approximation, which is well suited to describe the transverse dynamics of particle beams in plasmas. After the plasma the beam particles are propagated ballistically to the equivalent of the downstream OTR location, where beam transverse images and profiles can be generated and compared to the ones obtained experimentally. The 2-triangle fitting procedure is also applied to these images. The incoming $e^{+}$beam parameters are identical to those of the experiment. Detailed simulation parameters and results will be published elsewhere. Simulation results corresponding to the experimental results of Fig. 1 are displayed in Fig. 3(a) for $n_{e}=0$ and Fig. 3(b) for $n_{e}=$ $1.5 \times 10^{14} \mathrm{~cm}^{-3}$. These show remarkable similarities with the experimental beam images of Figs. 1(a) and 1(b). Also, both beam sizes and charge fractions in the core and halo of the beam [Figs. 3(c) and 3(d)] show good agreement with the experimental results of Figs. 2(a) and 2(b): similar beam sizes and dependencies in both planes, initial linear increase of the halo charge in the $y$ plane, and no significant halo formation in the $x$ plane. While the halo is fully formed at a higher density in the simulations than in the experiment (factor $2-3$ ), the asymptotic charge fractions in the halo are similar. This allows us to use the simulation results with confidence to study the effect of propagation through a plasma on the $e^{+}$beam.

In addition to the beam images, simulation results give access to the beam phase space and therefore to the beam emittance. Figure 4 shows the total $\mathrm{rms}$ bunch emittance as well as the emittance of five longitudinal or $z$ slices of the bunch, each containing $20 \%$ of the total charge, along the plasma length for $n_{e}=2 \times 10^{14} \mathrm{~cm}^{-3}$.
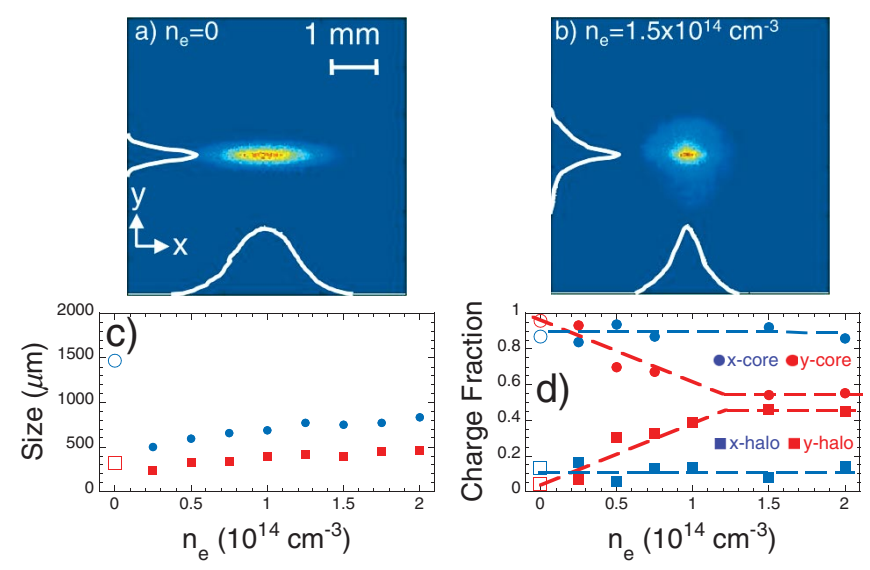

FIG. 3 (color). Simulated images of the beam at the downstream OTR location for (a) $n_{e}=0$ and (b) $n_{e}=1.5 \times$ $10^{14} \mathrm{~cm}^{-3}$; (c) beam transverse sizes ( $x$ plane, blue circles; $y$ plane, red squares); (d) fractions of the total beam charge in the beam core (circles) and the beam halo (square) in the $x$ (blue symbols) and $y$ plane (red symbols) as a function of $n_{e}$. These results are obtained from simulations with the experimental parameters. The empty symbols correspond to $n_{e}=0$. The dotted lines are plotted to suggest trends. 


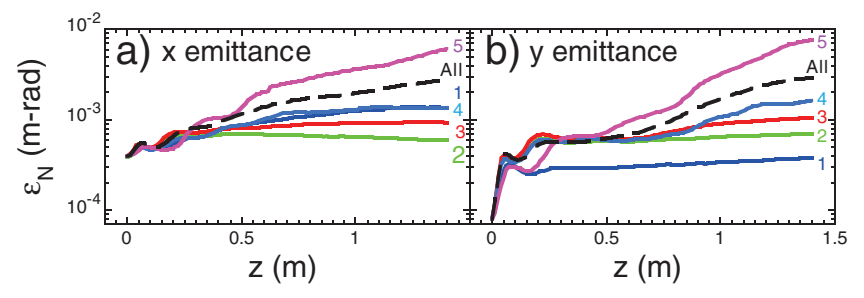

FIG. 4 (color). Emittance of the whole beam (dashed black line, labeled "All") and of five $z$ slices containing $20 \%$ each of the total beam charge, in the (a) $x$ and (b) $y$ plane as obtained from simulations. The lines are labeled with their slice number, starting from the bunch front. The focused beam size at the plasma entrance is $25 \mu \mathrm{m}$ (round); the incoming invariant emittances are $\epsilon_{N x} \approx 390 \times 10^{-6} \mathrm{~m} \mathrm{rad}$ and $\epsilon_{N y} \approx 80 \times$ $10^{-6} \mathrm{~m} \mathrm{rad}$, and $n_{e}=2 \times 10^{14} \mathrm{~cm}^{-3}$.

In the high emittance $x$ plane the emittance of all slices slowly increases as a function of distance. In contrast, in the low emittance $y$ plane, the emittance grows abruptly over the first few centimeters. The first four slices of the bunch exit the plasma with emittances in the 0.6-2.0 $\times$ $10^{-3} \mathrm{~m}$ rad range, smaller than the whole beam emittances (Fig. 4). The latter are dominated by the emittance growth in the last slice (number 5 in Fig. 4), where the onset of the two-stream hose instability $[24,25]$ is observed in the simulations at the largest values of $n_{e}$. It is interesting to note that, even though the incoming bunch emittances differ by a factor of about $5\left(\epsilon_{N x} \approx 5 \epsilon_{N y}\right)$, after $1.4 \mathrm{~m}$ of plasma they are about equal: $\epsilon_{N x} \approx \epsilon_{N y} \approx 3 \times 10^{-3} \mathrm{mrad}$. This corresponds to an emittance growth by factors of $\approx 8$ and $\approx 38$ in the two respective planes. However, the beam emittance may be closer to that of the core beam emittance, a factor two to three smaller (slices 1-4 only), since the quasistatic approximation may overestimate the growth of the hose instability observed in the last slice. The $e^{+}$bunch therefore exits the plasma with approximately equal emittances (Fig. 4) and sizes. Similar observations have been made in simulations of space charge dominated beams in an external focusing channel [26]. The dependency of the beam sizes on $n_{e}$, as seen in Figs. 2(a) and 3(a), suggests that the amount of emittance growth over a fixed plasma length increases with $n_{e}$. However, Fig. 4 indicates that at a given plasma density the emittance growth of the slices and of the total bunch level off after propagating some distance into the plasma. Numerical simulations of the $e^{+}$bunch propagating through a $n_{e}=1.5 \times 10^{14} \mathrm{~cm}^{-3}$ plasma with a length twice that of this experiment $(2.8 \mathrm{~m})$ show a further emittance growth by a factor of less than 2 . Some distance into the plasma (in simulations, $\approx 20 \mathrm{~cm}$ for $n_{e}=$ $1.5 \times 10^{14} \mathrm{~cm}^{-3}$ ), the $e^{+}$beam sizes and emittances, as well as the focusing force, reach essentially constant values; i.e., the beam can be propagated further without any more significant emittance growth.

We observed experimentally transverse beam size evolution accompanied by the formation of a charge halo after the propagation of a $e^{+}$beam through a 1.4-m-long plasma with a density in the $0.1-5 \times 10^{14} \mathrm{~cm}^{-3}$ range. Halo formation is the result of the nonlinear plasma focusing force. The observations are well described by numerical simulations. Simulations also show that, as expected, the beam emittance grows along the propagation. A possible option to mitigate the emittance growth suffered by an $e^{+}$bunch in a uniform plasma (this Letter) is to use a hollow channel plasma. In such an $e^{+}$PWFA, the radius $r_{c}$ of the hollow channel is on the order of the plasma collisionless skin depth $c / \omega_{p}$ [16]. Preliminary experimental results show that the distortions in beam transverse shape sustained by an $e^{+}$bunch along a low-density $\left(<10^{13} \mathrm{~cm}^{-3}\right), 1.4-\mathrm{m}-$ long hollow plasma channel are less than in the case of a uniform plasma [27]. Acceleration and emittance preservation of ultrashort $e^{+}$beams will be the subject of future experiments.

This work is supported by U.S. DOE Grants No. DEFG03-92ER40745, No. DE-AC02-76SF00515, No. DEFG03-98DP00211, and No. DEFG0392ER40727, and by NSF Grants No. ECS-9632735, No. DMS-9722121, and No. PHY-00787157.

[1] J. Stuckmeir et al., Particle Accelerators 15, 47 (1984).

[2] C. K. Allen et al., Phys. Rev. Lett. 89, 214802 (2002).

[3] C. Chen et al., Phys. Rev. Lett. 72, 2195 (1994).

[4] R. L. Gluckstern, Phys. Rev. Lett. 73, 1247 (1994).

[5] S. Bernal et al., Nucl. Instrum. Methods Phys. Res., Sect. A 519, 380 (2004).

[6] S. Lee et al., Phys. Rev. STAccel. Beams 5, 011001 (2002).

[7] I. Blumenfeld et al., Nature (London) 445, 741 (2007).

[8] J. B. Rosenzweig et al., Phys. Rev. A 44, R6189 (1991).

[9] C. E. Clayton et al., Phys. Rev. Lett. 88, 154801 (2002).

[10] N. Barov et al., Phys. Rev. Lett. 80, 81 (1998).

[11] J. Rosenzweig et al., Phys. Rev. Lett. 95, 195002 (2005).

[12] J. Ng et al., Phys. Rev. Lett. 87, 244801 (2001).

[13] M. Hogan et al., Phys. Rev. Lett. 90, 205002 (2003).

[14] P. Muggli et al., Phys. Rev. Lett. 93, 014802 (2004).

[15] C. O'Connell et al., Phys. Rev. ST Accel. Beams 5, 121301 (2002).

[16] S. Lee et al., Phys. Rev. E 61, 7014 (2000).

[17] B. Blue et al., Phys. Rev. Lett. 90, 214801 (2003).

[18] V. Balakin et al., Phys. Rev. Lett. 74, 2479 (1995).

[19] C. R. Vidal and J. Cooper, J. Appl. Phys. 40, 3370 (1969).

[20] P. Muggli et al., IEEE Trans. Plasma Sci. 27, 791 (1999).

[21] T. Wangler et al., in Proceedings of the $X X$ Linac Conference, Monterey CA, 2000, http://epaper.kek.jp/100/ papers/TU202.pdf, p. 341.

[22] J. Amundson et al., J. Comput. Phys. 211, 229 (2006).

[23] C. Huang et al., J. Comput. Phys. 217, 658 (2006).

[24] D. H. Whittum et al., Phys. Rev. Lett. 67, 991 (1991).

[25] E. S. Dodd et al., Phys. Rev. Lett. 88, 125001 (2002).

[26] R. A. Kishek, P. G. O'Shea, and M. Reiser, Phys. Rev. Lett. 85, 4514 (2000).

[27] K. A. Marsh et al., in Proceedings of the Particle Accelerator Conference, 2003, http://epaper.kek.jp/p03/ PAPERS/FOAB011.PDF, p. 731. 